\title{
Wide Stripe Texture of Liquid Crystalline Polymer after Cessation of Shear Flow*
}

\author{
Takehiro YAMAMOTO**, Kensuke OHNISHI* and Naoki MORIGUCHI ${ }^{* *}$ \\ ${ }^{* *}$ Department of Mechanical Engineering, Graduate School of Engineering, Osaka University \\ 2-1 Yamadaoka, Suita, Osaka 565-0871, Japan \\ E-mail: take@mech.eng.osaka-u.ac.jp
}

\begin{abstract}
Textures called wide stripe textures (WSTs) emerge after cessation of a simple shear flow of liquid crystalline polymers (LCPs). The present study considered the WST in a $50 \mathrm{wt} \%$ aqueous solution of hydroxypropylcellulose and investigated the distance between stripes. A test fluid was sandwiched between two parallel discs and shear was imposed on the fluid by rotating one of the discs. The experiments were carried out with varying the temperature of fluid, the shear rate $\dot{\gamma}$, and the gap height $h$. The distance tends to increase with increasing the gap width, slightly depends on $\dot{\gamma}$ for small $h$, and increases with increasing $\dot{\gamma}$ for large $h$. It was suggested that the emergence of WSTs related the relaxation process of relatively large structures such as defects in LCPs.
\end{abstract}

Key words : Liquid Crystalline Polymer, Shear Flow, Wide Stripe Texture, Hydroxypropylcellulose

\section{Introduction}

Liquid crystalline polymers (LCPs) are important engineering materials because of their properties such as high strength and rigidity, good moldability, and high thermal resistance. Liquid crystalline polymers are easily aligned by the flow and the orientation of molecules affects the strength of products made of LCPs. Consequently, the molecular orientation of LCPs in flows is an important subject and hence many researches have been carried out ${ }^{(1)}$.

The molecular alignment causes change in optical property of LCPs, and various kinds of textures are observed with an optical microscope. It is known that some textures appear both during simple shear flows of cholesteric LCPs and after cessation of the flow. Larson and $\mathrm{Mead}^{(2),(3)}$ have shown that striated textures parallel to the flow appears in simple shear flows. They concluded that the tumbling behavior of directors caused the texture. Navard ${ }^{(4)}$ reported the emergence of banded texture after shearing hydroxypropylcellulose (HPC) solutions and light-scattering patterns of textures were investigated. Viney and Putnam ${ }^{(5)}$ have studied banded textures that appear after cessation of simple shear flow of HPC solutions. The mechanism of the emergence of banded textures was explained as follows: In general, LCP molecules tend to be aligned in the flow direction. When the shear flow stops, the molecules change their orientations to release elastic strains and form a zig-zag structure, which is observed as a banded texture under crossed polarizers.

Mori et al. ${ }^{(6)}$ have investigated the texture of HPCs after cessation of simple shear flows and found that a wide stripe texture (WST) appeared in a long period after the disappearance of banded structures. In this study, the condition for the emergence of WSTs was investigated and it was reported that the WST was observed at moderate to high shear rates and for relatively large gap heights. In addition, they carried out SALS (small angle light scattering) experiments using $\mathrm{He}-\mathrm{Ne}$ laser and confirmed that scattering patterns became circular during a period when a WST is observed ${ }^{(6),(7)}$. This fact means that structures of the order of $1 \mu \mathrm{m}$, which is the order of the size of a scatterer, disappear and a WST consists of larger scale 


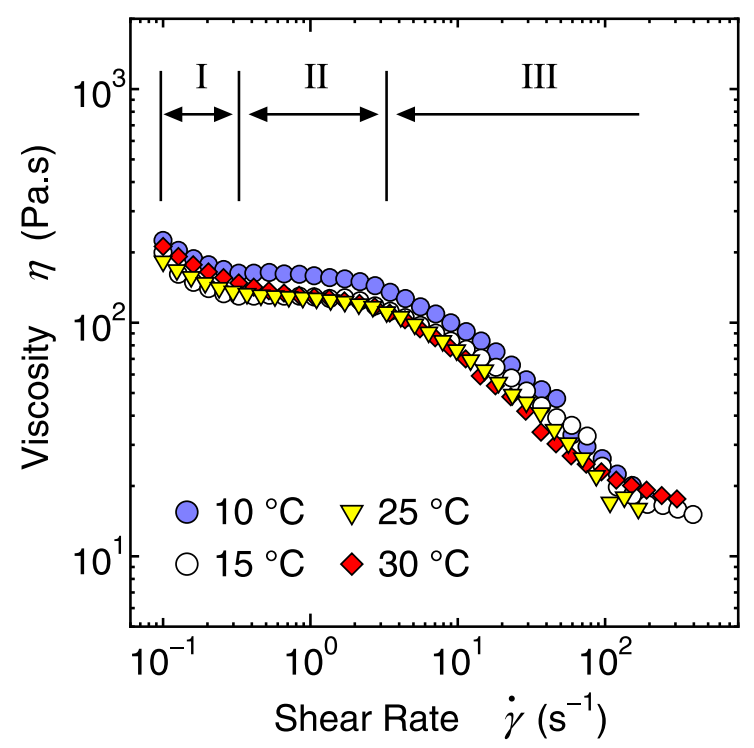

Fig. 1 Steady shear viscosity of the $50 \mathrm{wt} \%$ aqueous solution of HPC at temperatures $T=10,15,25$, and $30^{\circ} \mathrm{C}$. The symbols I, II, III indicate the shear rate ranges of Region I, Region II, and Region III, respectively.

structures.

In the present study, for quantitative analyses of WST, we focused on the distance between stripes in WSTs and investigated the dependence of the distance on temperature, a gap height, and the shear rate. LCPs under shear flows between parallel plates were observed with an optical microscope.

\section{Test fluids}

The test fluid is a $50 \mathrm{wt} \%$ aqueous solution of HPC (HPC-L supplied by Nippon Soda). Aqueous solutions of the HPC are cholesteric LCPs at the concentration above about $42 \mathrm{wt} \%$. Consequently, the test fluid is in a cholesteric phase. Figs. 1 and 2 show the steady shear viscosity $\eta$ and the first normal stress difference $N_{1}$ of the test fluid, which were measured with a cone-plate-type rheometer (Physica MCR300 manufactured by Anton Paar). In Fig. 1 , the shear viscosity has three regions, which are the first shear-thinning region at low shear rates (Region I), a plateau region at moderate shear rates (Region II), and the second shearthinning region at high shear rates (Region III). In Region I, polydomain structures of LCP collapse and viscosity decreases with increasing the shear rate. The secondary shear-thinning in Region III is due to the molecular alignment caused by the shear flow.

In Fig. 2 at high shear rates, $N_{1}$ is negative as reported in previous studies ${ }^{(4),(8)}$. The first normal stress difference turns from positive to negative when the motion of directors changes from the tumbling to the wagging ${ }^{(9)}$. The critical shear rate is estimated as the shear rate where $N_{1}$ is zero and is higher at lower temperature as shown in Fig. 2. It is understood that the value of $N_{1}$ takes a negative local minimum at high shear rates and the corresponding shear rate is a critical value of transition from the wagging region and the aligning one ${ }^{(9)}$, which was not observed in the measurement region in Fig. 2.

\section{Experimental setup and method}

The shear is imposed on a test fluid using a Cambridge Shearing System, CSS-450 manufactured by Linkam Scientific Instrument Ltd. Fig. 3 shows the schematic of the system. The test fluid is sandwiched between two highly polished quartz plates and each plate is in thermalcontact with an independently controlled pure silver. The temperature is controlled within a range of $\pm 0.2^{\circ} \mathrm{C}$. One of the plates rotates and the other is fixed, and hence the shear is imposed on the fluid. The center of observation window locates $7.5 \mathrm{~mm}$ from the rotation 


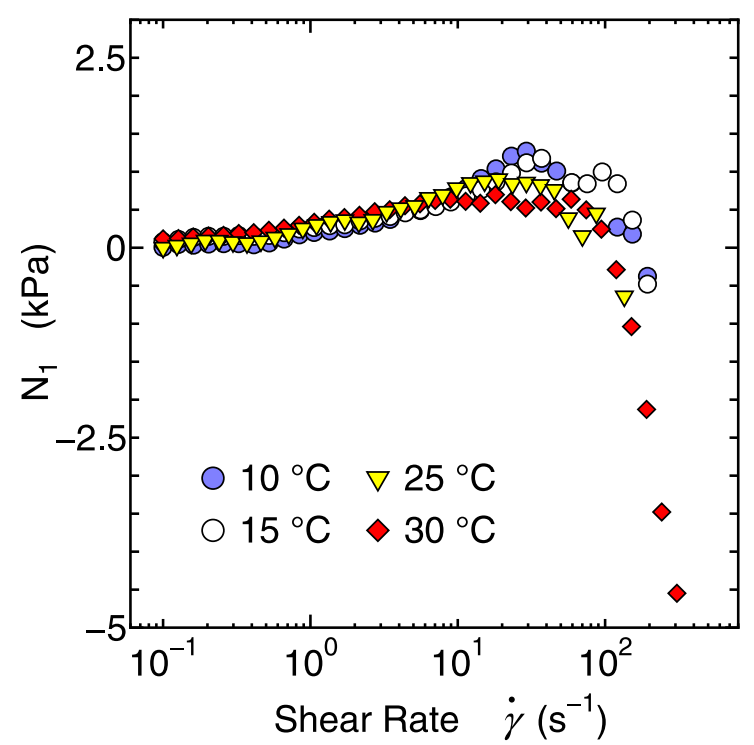

Fig. 2 The first normal stress difference $N_{1}$ of the $50 \mathrm{wt} \%$ aqueous solution of HPC at temperatures $T=10,15,25$, and $30^{\circ} \mathrm{C}$.

axis and the observation window is $2.5 \mathrm{~mm}$ in diameter. The shear rate is calculated by $r \omega / h$, where the radial position $r=7.5 \mathrm{~mm}, \omega$ is angular velocity, and $h$ is the gap height between the plates.

The texture of LCPs is observed with an optical microscope under crossed polarizers (Fig. 4). In addition, a quarter-wave plate is inserted between the polarizers to obtain colored images of texture, which are helpful for the evaluation of the distance between stripes. Change in the texture with time is recorded with a digital video recorder connected to the microscope.

Here, we explain the experimental procedure. As shown in Fig. 5, a test fluid between two plates is pre-sheared at the shear rate of $0.1 \mathrm{~s}^{-1}$ for $120 \mathrm{~s}$ and is left at rest for $120 \mathrm{~s}$. These processes are necessary for preparing an equivalent molecular orientation state for each observation. Next, a shear at a specific shear rate $\dot{\gamma}$ is imposed for $120 \mathrm{~s}$, and the shear is stopped at the time $t=0$. That is, $t$ indicates the elapsed time after cessation of shear.

The experiments were carried out for various temperatures $T$, gap heights between the plates $h$, and shear rates $\dot{\gamma}$. The behavior of WSTs was analyzed under the conditions summarized in Table 1, which were chosen from conditions under which WSTs emerge. Color information is extracted from digital images of textures and is analyzed using the maximum entropy method (MEM) to obtain the distance between the stripes.

\section{Results and discussion}

Figure 6 shows typical textures observed. The experimental condition is $h=500 \mu \mathrm{m}, \dot{\gamma}=10$ $\mathrm{s}^{-1}$, and $T=25^{\circ} \mathrm{C}$. Before the imposition of shear, the texture is featureless at equilibrium as shown in Fig. 6(a). A stripe texture appears under the shear flow and the long side of texture is parallel to the flow direction (Fig. 6(b)). This texture is observed during the flow. Just after cessation of shear, the texture begins to fade away and a different kind of texture appears (Figs. 6(a)-(d)). The newly appeared texture is called a banded texture, whose long side is perpendicular to the flow. At $t=10 \mathrm{~s}$, the stripe texture perfectly disappears and only the banded texture is seen (Fig. 6(e)). The texture becomes featureless once again (Fig. 6(f)) and then a wide stripe texture (WST) appears as shown in Figs. 6(g)-(i).

During a shear flow, striated textures parallel to the flow direction appear owing to the director orientation. After cessation of the flow, with relaxing the molecular orientation, the striated textures disappear and stripe textures emerge. The molecules change their orientations to release elastic strains caused by the flow and form a zig-zag structure. This structure is 


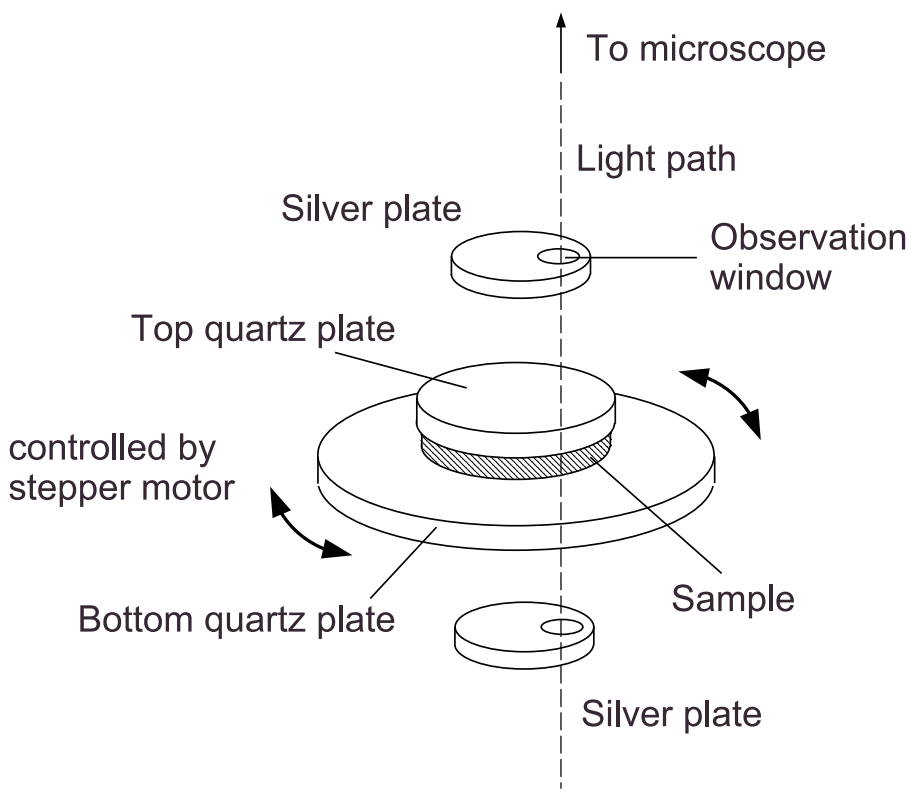

Fig. 3 Schematic of shearing system.

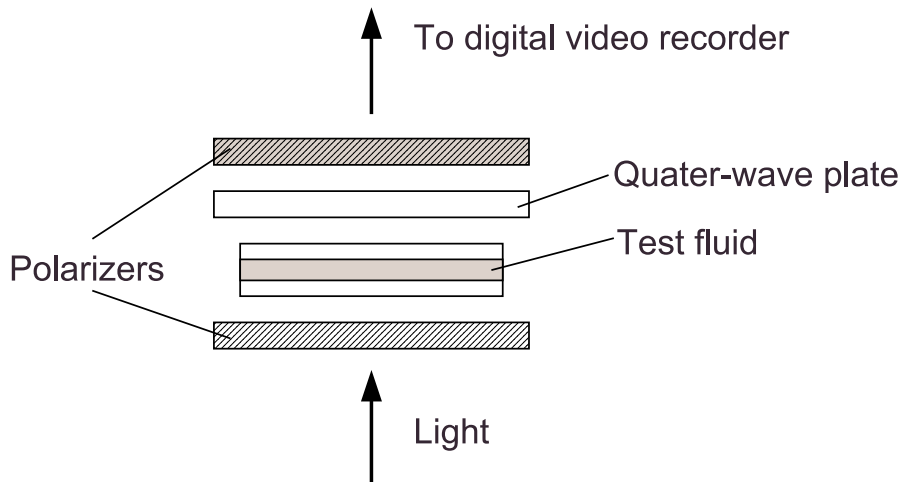

Fig. 4 Schematic of optical system.

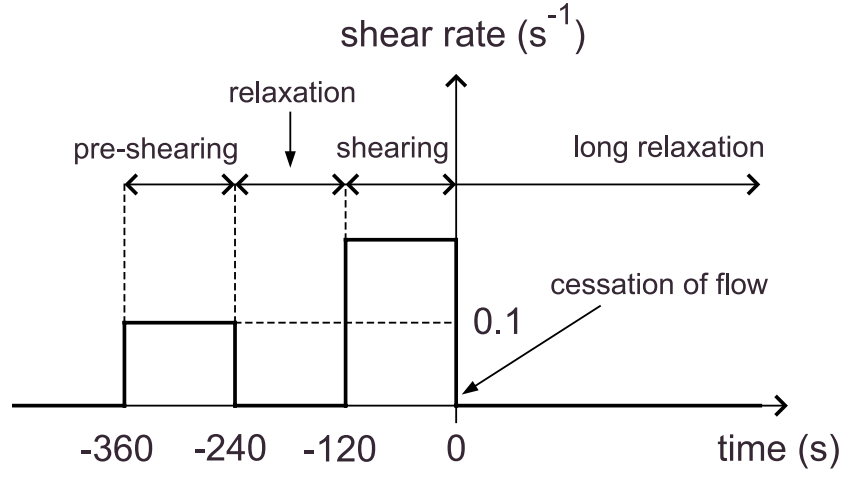

Fig. 5 Schematic of experimental procedure.

Table 1 Experimental condition

\begin{tabular}{c|c|c}
\hline Temperature $T\left({ }^{\circ} \mathrm{C}\right)$ & Gap height $h(\mu \mathrm{m})$ & Shear rate $\dot{\gamma}\left(\mathrm{s}^{-1}\right)$ \\
\hline $10,15,25,30$ & $200,300,400,500$ & $3.0,5.0,7.0,10$ \\
\hline
\end{tabular}




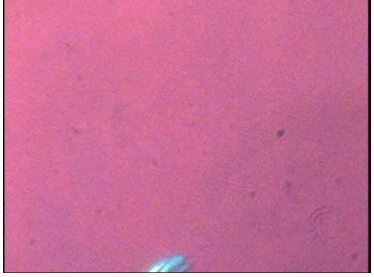

(a)

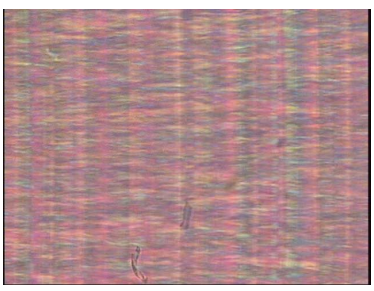

(d)

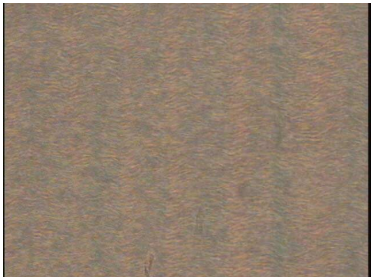

(g)

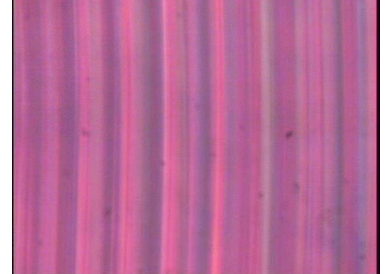

(b)

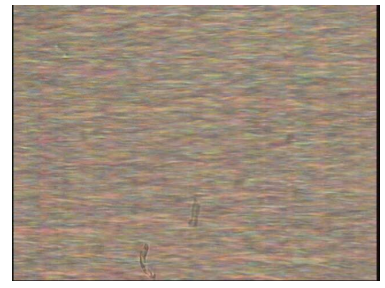

(e)

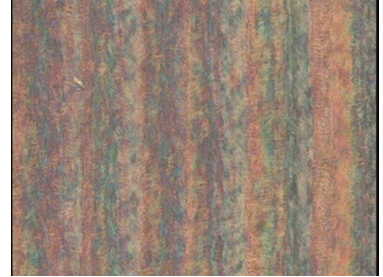

(h)

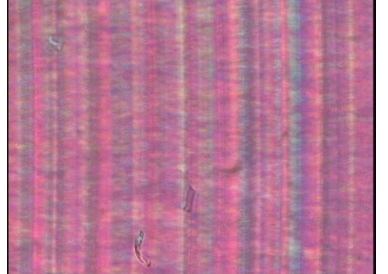

(c)

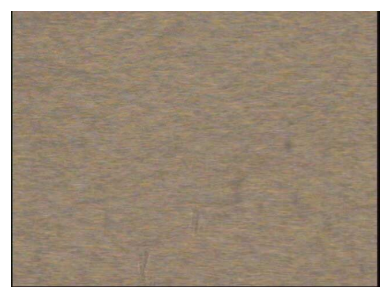

(f)

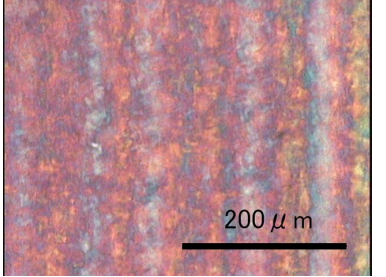

(i)

Fig. 6 Examples of typical textures observed both in a shear flow of the $50 \mathrm{wt} \%$ aqueous solution of HPC and after cessation of the flow. The flow direction is from bottom to top: $h=500 \mu \mathrm{m}, \dot{\gamma}=10 \mathrm{~s}^{-1}, T=25^{\circ} \mathrm{C}$. $t=(\mathrm{a})-120 \mathrm{~s}$, (b) $-60 \mathrm{~s}$, (c) $0 \mathrm{~s}$, (d) $5 \mathrm{~s}$, (e) $10 \mathrm{~s}$, (f) $50 \mathrm{~s}$, (g) $100 \mathrm{~s}$, (h) $150 \mathrm{~s}$, and (i) $360 \mathrm{~s}$.

observed as a banded texture perpendicular to the flow direction under crossed polarizers ${ }^{(5)}$. As the molecular orientation relaxes, the banded structure disappears . In WSTs that emerges in a long period after the disappearance of banded textures, the long side of stripe is parallel to the flow direction similarly to the striated texture observed during shear flows. This fact suggests that some kind of memory effect in molecular orientation exists.

The condition for the emergence of WST has been investigated for wide ranges of $h$ and $\dot{\gamma}$, and it was reported that WSTs emerged at moderate shear rates and relatively large $h^{(6),(7)}$. We focused our efforts on experiments under conditions where the WST emerges. Thus, the range of $\dot{\gamma}$ in the present experiments is $3.0-10 \mathrm{~s}^{-1}$ and the range of gap height is $200-500$ $\mu \mathrm{m}$. The condition for the emergence of WST is summarized in Fig. 7. We can see that WSTs appeared at small shear rates for small gap sizes and they are seen also at relatively high shear rates for large gap sizes.

We next analyze the dependence of the distance between stripes $d_{s}$ on temperature $T$, the gap height $h$, and the shear rate $\dot{\gamma}$. Figure 8 explains the procedure for evaluating $d_{s}$. Firstly, the color information of each image is scanned along a line perpendicular to the flow direction (Fig. 8(a)). Then, the color information of a texture is converted to the color number (Fig. 8(b)). Finally, the converted data are analyzed by MEM and a distance between stripes is calculated using a peak frequency (Fig. 8(c)). This procedure is repeated for another line and $d_{s}$ is evaluated as the average of two values of the distance.

Figure 9 plots $d_{s}$ as a function of the shear rate for various $h$ and $T$. The graph is separately drawn for each temperature. Temperature does not affect $d_{s}$ significantly within the range of $T$ in the present experiments because the HPC is in a cholesteric phase at each temperature and rheological properties such as $\eta$ and $N_{1}$ in Figs. 1 and 2 slightly depend on the temperature. In Fig. 9, we can see general tendencies that the distance between stripes is larger for larger $h$, does not depend on the shear rate for small $h$, and increases with increasing the shear rate for large $h$. Since $d_{s}$ depends on $h$, the WST relates structures of much larger 


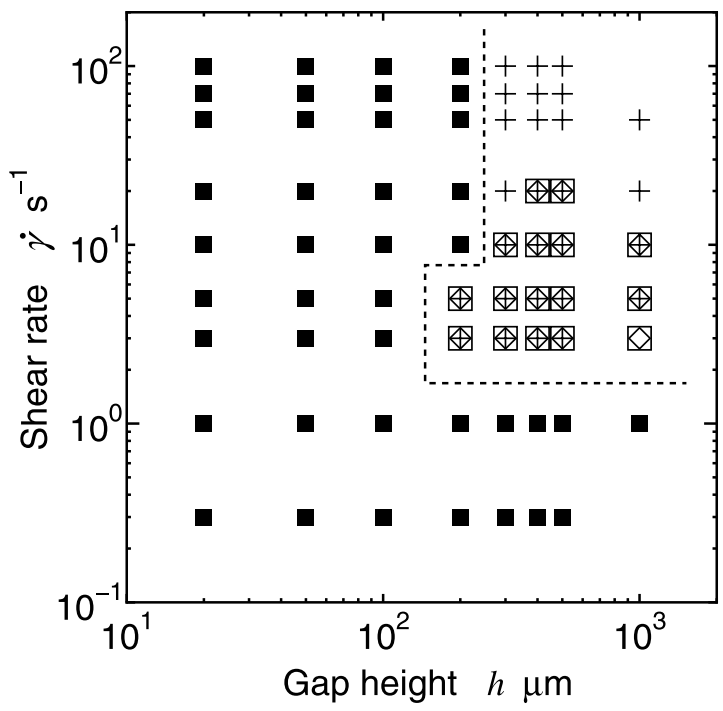

Fig. 7 Emergence map of wide stripe texture (WST): $\mathbf{n}$, WST is not observed; The symbols $\square, \diamond$, and + indicate that WSTs emerge under those conditions and correspond to data at $10{ }^{\circ} \mathrm{C}, 25^{\circ} \mathrm{C}$, and $30{ }^{\circ} \mathrm{C}$, respectively.

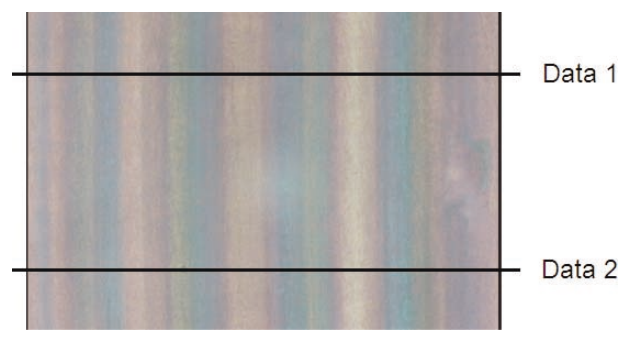

(a)

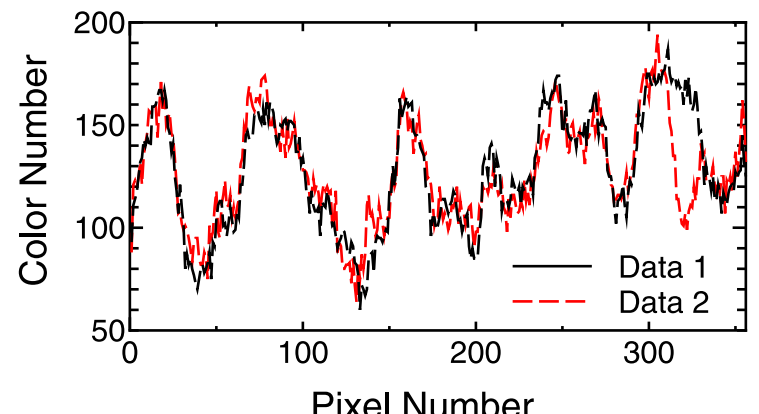

(b)

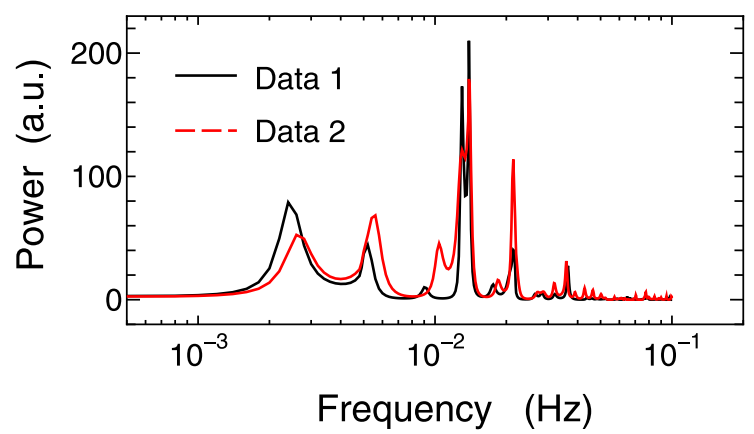

(c)

Fig. 8 Example of the procedure for evaluating $d_{s}$ : (a) image data, (b) transformation of image data to color number data, (c) MEM analysis of data of (b). 


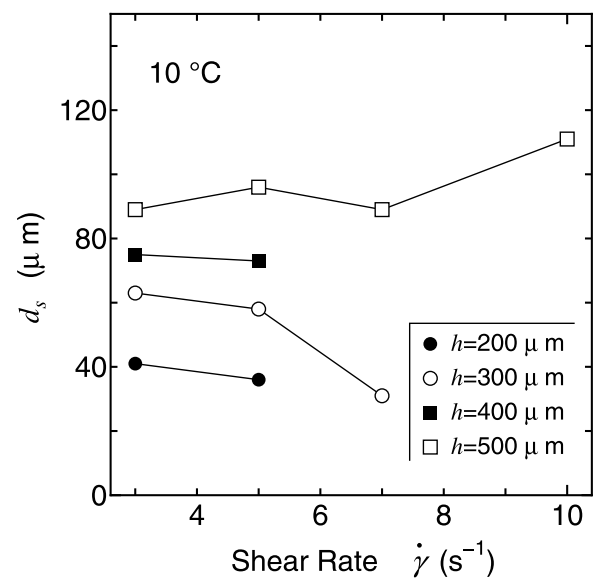

(a)

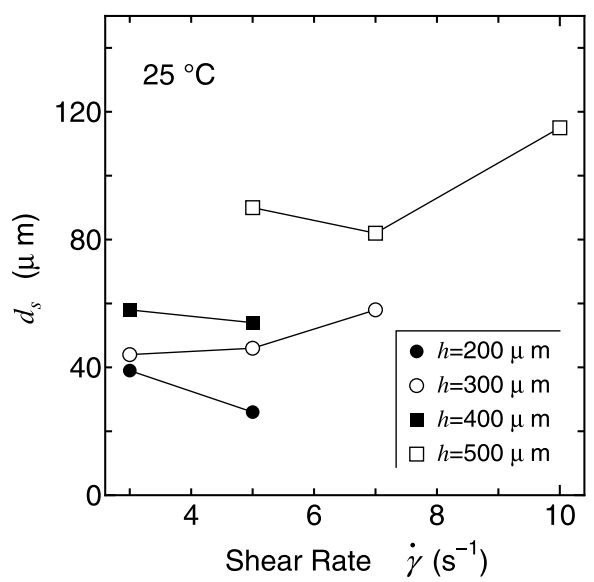

(c)

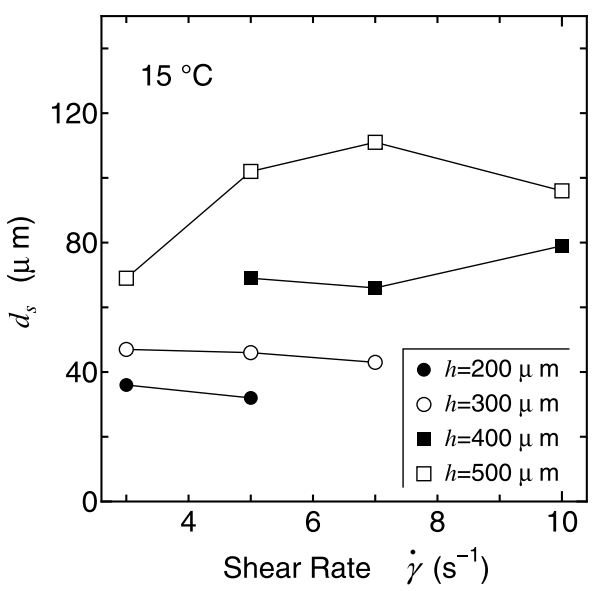

(b)

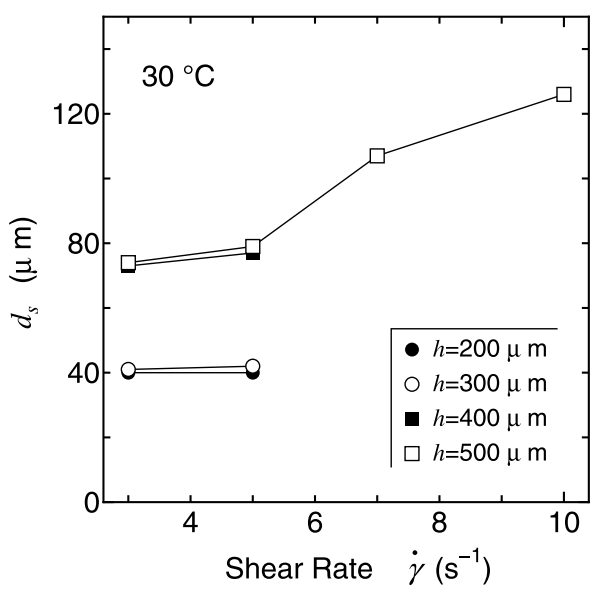

(d)

Fig. 9 Dependence of distance between stripes $d_{s}$ on shear rate $\dot{\gamma}$ at $T=\left(\right.$ a) $10^{\circ} \mathrm{C}$, (b) $15^{\circ} \mathrm{C}$, (c) $25^{\circ} \mathrm{C}$, (d) $30^{\circ} \mathrm{C}$.

scale. In addition, previous SALS measurements ${ }^{(6),(7)}$ indicate that the WST consists of structures larger than the order of $1 \mu \mathrm{m}$, and these results of $h$-dependence of $d_{s}$ suggest that the WST relates relatively large structures.

In the present study, we also carried out SALS experiments to investigate the scattered light intensity through a test fluid. In the experiments, a He-Ne laser beam was transmitted through the observation window and the intensity of scattered light was measured. Figure 10 shows change in scattered light intensity $I$ scaled by the initial light intensity $I_{0}$ with time at $\dot{\gamma}=10 \mathrm{~s}^{-1}$ and $T=25^{\circ} \mathrm{C}$ for $h=500 \mu \mathrm{m}$. The light intensity quickly increases when a banded texture emerges, saturates, and begins to decrease with time. The decrease in $I / I_{0}$ has two stages and the decreasing rate changes around $t=70 \mathrm{~s}$, which corresponds to a region of a circle in Fig. 10. This period coincides with the emergence time of WSTs and the second stage of decrease in the light intensity was not observed when WSTs do not emerge.

Under shear, molecules tend to be aligned in the flow direction and hence directors orient to the flow direction. At high shear rates, the molecules significantly orient, and highly oriented region and a low density of local defects coexists. After cessation of shear flow, the elastic energy stored in local defects during the shear becomes a driving force to rotate directors $^{(5)}$. Consequently, a zig-zag structure (Fig. 11) is formed and is observed as a banded structure under crossed polarizers. This relaxation process immediately occurs on cessation of shear and hence the banded texture emerges just after cessation of flow. If scatterers with a periodic structure exist, the intensity of scattered light increases. The quick increases in $I / I_{0}$ 


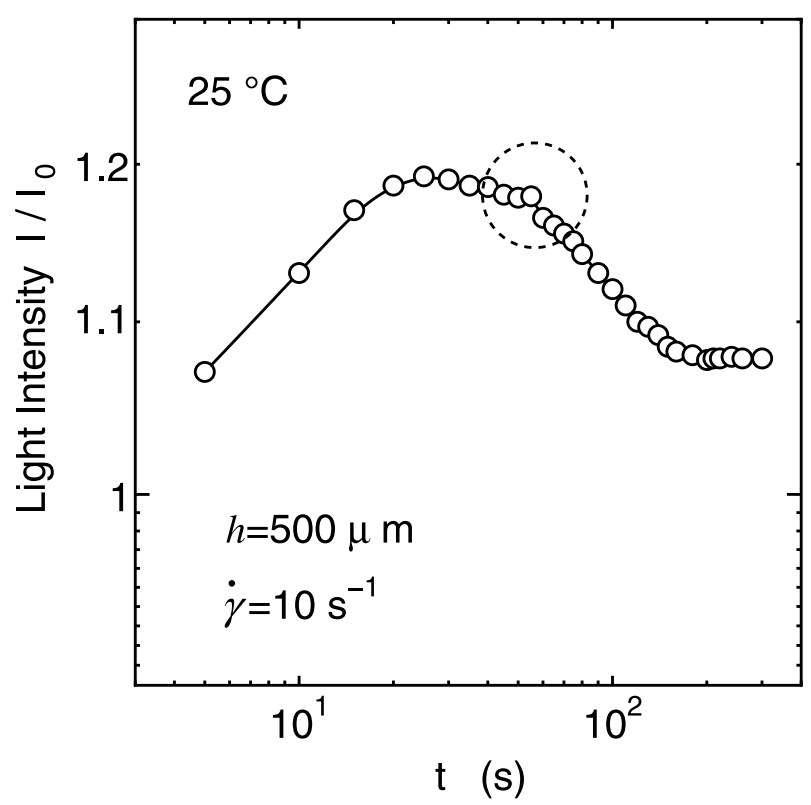

Fig. 10 Change in light intensity with time at $\dot{\gamma}=10 \mathrm{~s}^{-1}$ and $T=25^{\circ} \mathrm{C}$ for $h=500 \mu \mathrm{m}$.

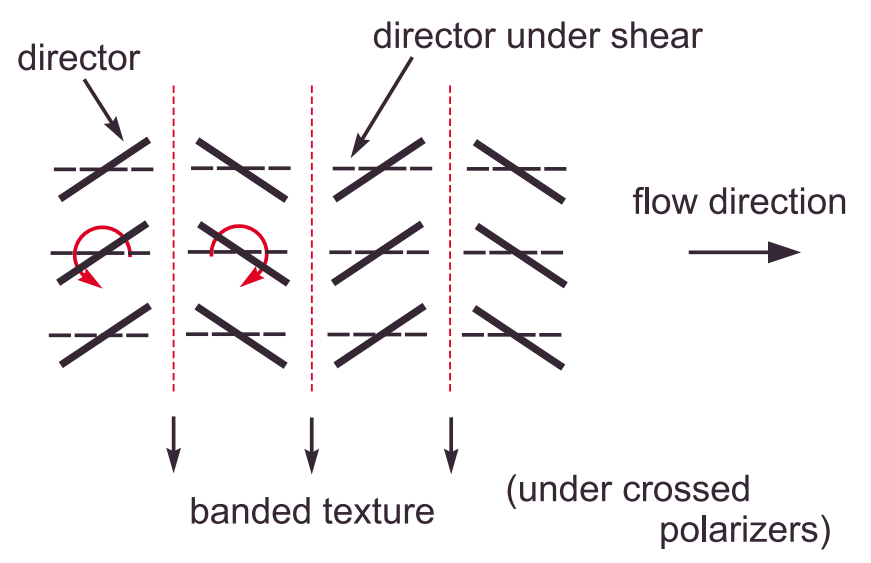

Fig. 11 Schematic of emergence of zig-zag structure after cessation of shear flow. Thick solid segments indicate directors after cessation of shear flow and dashed segments indicate directors in shear flow.

corresponds to this property. As the relaxation of molecular orientation proceeds, the zig-zag structure fades away and the banded texture disappears. The light intensity may have upper bound and hence $I / I_{0}$ saturates and finally begins to decrease as the relaxation proceeds.

In Fig. 10, we can recognize that two types of relaxation processes exist after cessation of shear flow. The first relaxation corresponds to the director relaxation to form banded textures, which is explained above. The second one appears at a time similar to the emergence time of WSTs. WSTs are concealed as long as a banded texture is seen. They finally become visible in a long period after the disappearance of banded texture.

The structural change in WSTs has not been directly captured in the present texture observation. However, it is available to propose possible mechanism of the emergence of WSTs from the present experimental results. We think that the emergence of WST relates a long-time relaxation process of relatively large scale structure such as defects along the flow direction caused by shear at relatively high shear rates. The experimental results are consistent with this idea. WSTs, whose long side is parallel to the flow direction, do not emerge at small shear rates or at very high shear rates; a defect does not appear at small shear rates and 
collapses at very high shear rates. Furthermore, WSTs are not observed for small gap height, which means spatial constrain exists for the emergence of WSTs. SALS experiments ${ }^{(6),(7)}$ also support an idea that WSTs originate a relatively large scale inner structure. Thus, it is reasonable to consider that the emergence of WST relates the relaxation of defects. However, information of fluid inner structures will be necessary to confirm the structural change that relates the emergence of WSTs.

\section{Conclusion}

We studied wide stripe textures that emerge after cessation of a shear flow of HPC solutions. The behavior of WST was observed with an optical microscope under crossed polarizers and the distance between stripes $d_{s}$ was evaluated. Furthermore, we carried out SALS experiments to investigate change in the density of scattered light through a test fluid when a texture evolves. The results obtained are as follows:

(1) WSTs emerge long after the disappearance of a banded texture that begins to emerge just after cessation of shear flow. It is confirmed that WSTs emerge under conditions of relatively large shear rates $\dot{\gamma}$ and wide gap height $h$.

(2) In general, $d_{s}$ is larger for $h$, does not depend on $\dot{\gamma}$ for small $h$, and increases with increasing $\dot{\gamma}$ for large $h$.

(3) Behavior of decrease in the intensity of scattered light through a test fluid changes around the emergence of WST. This fact indicates that the emergence of WST relates a longtime relaxation process of relatively large structure.

The mechanism of the emergence of WSTs or the origin of the dependence of $d_{s}$ on both $h$ and $\dot{\gamma}$ has not been clarified yet. In addition, optical measurements with $\operatorname{SALS}^{(6),(7)}$ did not capture structural change in WSTs. However, present experimental results suggest that the emergence of WSTs relates the relaxation of relatively large structures and the defect in a fluid is a possibility. In future study, it may be necessary to analyze the dynamics of fluid inner structure, and the numerical simulation of three-dimensional orientation behavior of LCPs in a shear flow and of the relaxation process after cessation of the shear flow will help to clarify these problems.

\section{References}

( 1 ) Rey, A.D. and Denn, M.M., Dynamical Phenomena in Liquid-Crystalline Materials. Annual Review of Fluid Mechanics, Vol.34 (2002), pp.233-266.

( 2 ) Larson, R.G. and Mead, D.W., Development of Orientation and Texture During Shearing of Liquid-Crystalline Polymers, Liquid Crystal, Vol.12, No.5 (1992), pp.751-768.

( 3 ) Larson, R.G. and Mead, D.W., The Ericksen Number and the Deborah Number Cascades in Sheared Polymeric Nematics, Liquid Crystal, Vol.15, No.2 (1993), pp.151-169.

( 4 ) Navard, P., Formation of Band Textures in Hydroxypropylcellulose Liquid Crystals, Journal of Polymer Science: Polymer Physics Edition, Vol.24, No.2 (1986), pp.435442.

( 5 ) Viney, C. and Putnam, W.S., The Banded Microstructure of Sheared Liquid-Crystalline Polymers, Polymer, Vol.36, No.9 (1995), pp.1731-1741.

( 6 ) Mori, N. Moriguchi, N., Yamamoto, T. and Yasuda, K., Textures Observed in a Simple Shear Flow and After Cessation of the Flow for Liquid Crystalline Polymers, Slow Dynamics in Complex Systems, AIP Conference Proceedings, Vol.708 (2004), pp.96-97.

( 7 ) Moriguchi N., Texture Formation in Liquid Crystalline Polymers in Shear Flows and After Cessation of the Flow, Master Thesis of Osaka University (2005) (in Japanese).

( 8 ) Larson, R.G., Arrested Tumbling in Shearing Flows of Liquid Crystalline Polymers, Macromolecules, Vol.23, No.17 (1990), pp.3983-3992.

( 9 ) Burgharadt, W.R., Macromolecular Chemistry and Physics, Vol.199, No.4 (1998), pp.471-488. 